\title{
An Upper Bound for Conditional Second Moment of the Solution of a SDE
}

\author{
Andriy Yurachkivsky \\ Cybernetics Department, Taras Shevchenko National University, Kyiv, Ukraine \\ Email: andriy.yurachkivsky@gmail.com
}

Received November 9, 2012; revised December 9, 2012; accepted December 16, 2012

\section{ABSTRACT}

Let $\mathbb{F}=\left(\mathcal{F}(t), t \in \mathbb{R}_{+}\right)$be a filtration on some probability space and let $\mathcal{K}$ denote the class of all $\mathbb{F}$-adapted $\mathbb{R}^{d}$-valued stochastic processes $M$ such that $\mathrm{E}\left(|M(t)|^{2} \mid \mathcal{F}(0)\right)<\infty, \mathrm{E}(M(t) \mid \mathcal{F}(s))=M(s)$ for all $t>s \geq 0$ and the process $\mathrm{E}\left(|M(\cdot)|^{2} \mid \mathcal{F}(0)\right)$ is continuous (the conditional expectations are extended, so we do not demand that $\left.\mathrm{E}|M(t)|^{2}<\infty\right)$. It is shown that each $M \in \mathcal{K}$ is a locally square integrable martingale w. r. t. $\mathbb{F}$. Let $X$ be the strong solution of the equation $X(t)=\int_{0}^{t} Q(s, X(s)) \mathrm{d} l(s)+M(t)$, where $M \in \mathcal{K}, \quad l$ is a continuous increasing process with $\mathcal{F}(0)$-measurable values at all times, and $Q$ is an $\mathbb{R}^{d}$-valued random function on $\mathbb{R}_{+} \times \mathbb{R}^{d}$, continuous in $x \in \mathbb{R}^{d}$ and $\mathbb{F}$-progressive at fixed $x$. Suppose also that there exists an $\mathcal{F}(0) \otimes \mathcal{B}_{+}$-measurable in $(\omega, t)$ nonnegative random process $\psi$ such that, for all $t, x, x^{\mathrm{T}} Q(t, x) \leq-\psi(t)|x|^{2}$ and $\int_{0}^{t} \psi(s) \mathrm{d} t(s)<\infty$. Then $\mathrm{E}^{0}|X(t)|^{2} \leq \mathrm{e}^{-\Psi(t)}|M(0)|^{2}+\mathrm{e}^{-\Psi(t)} \int_{0}^{t} \mathrm{e}^{\Psi(s)} \mathrm{dE}^{0} \operatorname{tr}\langle M\rangle(s)$, where $\Psi(t)=2 \int_{0}^{t} \psi(\tau) \mathrm{d} t(\tau)$.

Keywords: Conditional Expectation; Martingale; Stochastic Equation

\section{Introduction}

The random processes under consideration are assumed, firstly, given on a common probability space $(\Omega, \mathcal{F}, \mathrm{P})$ (without any exception) and, secondly, càdlàg (the exceptions will be stipulated). Let $\mathcal{F}^{0}$ be a sub- $\sigma$ algebra of $\mathcal{F}$. We introduce the notation:

$\mathrm{E}^{0}=\mathrm{E}\left(\cdots \mid \mathcal{F}^{0}\right), \mathrm{P}^{0}=\mathrm{P}\left\{\cdots \mid \mathcal{F}^{0}\right\} ; \mathcal{V}_{0}^{+}$- the class of all increasing from zero numeral random processes whose values at all times are $\mathcal{F}^{0}$-measurable random variables. If, besides, a filtration $\mathbb{F}=\left(\mathcal{F}(t), t \in \mathbb{R}_{+}\right)$is given, then we identify $\mathcal{F}^{0}$ with $\mathcal{F}(0)$. By $\overline{\mathcal{M}}_{2}$ we denote, following [1], the class of all $\mathbb{R}^{d}$-valued ( $d$ will be determined by the context, if matters) $\mathbb{F}$-martingales $M$ such that for every $t \mathrm{E}|M(t)|^{2}<\infty ; \ell \mathcal{M}_{2}$ signifies (see ibid.) the class of all locally square integrable martingales w. r. t. $\mathbb{F}$.

The definition of conditional expectation, in particular $\mathrm{E}^{0}$, adopted in this article is due to Meyer (see [2]). It admits existence of the conditional expectation of a random variable with infinite first absolute moment.
Thus generalized conditional expectation inherits most of the familiar properties (listed, for example, in [2]) of the classical one, but in this case new proofs are required. They are gathered in Section 2.

Let $X$ be the solution of a stochastic differential equation of the kind

$$
X(t)=\int_{0}^{t} Q(s, X(s)) \mathrm{d} \imath(s)+M(t),
$$

where $l$ is a continuous process from $\mathcal{V}_{0}^{+}$and $M$ is chosen from some subclass of $\ell \mathcal{M}_{2}$ which is constructed and studied in Section 3. The goal of this article is to find an upper bound, much more exact than that provided by the Gronwall-Bellman lemma, for $\mathrm{E}^{0}|X(t)|^{2}$. This is done in Section 4 containing the only final result of the article. The reader inclined to accept that result in less generality, when $M$ is a quasicontinuous process from $\overline{\mathcal{M}}_{2}$ and $\mathcal{F}^{0}=\{\varnothing, \Omega\}$ (so that $\mathrm{E}^{0}=\mathrm{E}$ ), may skip all the preceding material. But for the approach underlying the derivations in Section 4 such a confinement is unnatural. That is a reason why $3 / 4$ of the 
article's volume are allocated to ancillary results. Another reason is that those results may prove useful beyond the context of this article.

Upper bounds for $\mathrm{E}|X(t)|^{p}$ are usually obtained with the aid of Lyapunov's functions (see, e.g., [3,4]). Our alternative approach is based on a "comparison theorem" (Corollary 4.2) allowing both to weaken the assumptions and to refine the conclusion (cf. our Theorem 4.3 with Theorem I.4.2 in [3]).

All vectors are thought of, unless otherwise stated, as columns; $\int_{a}^{b}$ means $\int_{] a, b]}$. The space of all $d$-dimensional row vectors with real components is denoted $\mathbb{R}^{d *}$. The words "almost surely" are tacitly implied in relations between random variables, including the convergence relation, unless it is explicitly written as the convergence in probability. Indicators are denoted by $I$ with two possible modes of writing the set: $I_{B}$ or $I\{\cdots\}$.

The reference books for the notions and results of stochastic analysis used in this paper are $[1,5,6]$.

\section{Extended Conditional Expectations}

Denote $\overline{\mathbb{R}}_{+}=\mathbb{R}_{+} \cup\{\infty\}$ and, for $a \in \mathbb{R}, \quad a_{+}=a \vee 0, a_{-}=-(a \wedge 0)$, so that $a=a_{+}-a_{-}$. In what follows, "nonnegative" means " $\mathbb{R}_{+}$-valued" (the value $\infty$ is not admitted). The Borel $\sigma$-algebra in $\mathbb{R}_{+}$ will be denoted $\mathcal{B}_{+}$.

Let $\mathcal{G}$ be a sub- $\sigma$-algebra of $\mathcal{F}$. The conditional given $\mathcal{G}$ expectation of an $\overline{\mathbb{R}}_{+}$-valued random variable $\underline{\gamma}$ is defined, according to [2], as the $\mathcal{G}$-measurable $\overline{\mathbb{R}}_{+}$-valued random variable $\mathrm{E}(\gamma \mid \mathcal{G})$ such that

$\mathrm{E} \gamma I_{G}=\mathrm{E}\left(\mathrm{E}(\gamma \mid \mathcal{G}) I_{G}\right)$ for every $G \in \mathcal{G}$. For an $\mathbb{R}$ valued random variable $\gamma$ such that $\mathrm{P}\left\{\mathrm{E}\left(\gamma_{+} \mid \mathcal{G}\right)=\infty=\mathrm{E}\left(\gamma_{-} \mid \mathcal{G}\right)\right\}=0$ we set by definition $\mathrm{E}(\gamma \mid \mathcal{G})=\mathrm{E}\left(\gamma_{+} \mid \mathcal{G}\right)-\mathrm{E}\left(\gamma_{-} \mid \mathcal{G}\right)$. Further the conditional expectation of a $\mathbb{C}^{d}$-valued random variable is defined in the obvious way. Thus defined conditional expectation will be called extended. Unlike the classical conditional expectation (defined only for $\gamma \in \mathrm{L}_{1}(\Omega, \mathcal{F}, \mathrm{P})$ ) it does not possess, generally speaking, the property

$$
\mathcal{G}_{1} \subset \mathcal{G}_{2} \Rightarrow \mathrm{E}\left(\mathrm{E}\left(\gamma \mid \mathcal{G}_{2}\right) \mid \mathcal{G}_{1}\right)=\mathrm{E}\left(\gamma \mid \mathcal{G}_{1}\right) .
$$

But for an $\overline{\mathbb{R}}_{+}$-valued $\gamma$ this property remains valid - with the same proof as for $\gamma \in \mathrm{L}_{1}$.

Obviously, the extended conditional expectation of $\beta \in \mathrm{L}_{1}(\Omega, \mathcal{F}, \mathrm{P})$ coincides with the classical one and therefore

$$
\begin{aligned}
\mathrm{E}(\beta+\gamma \mid \mathcal{G}) & =\mathrm{E}(\beta \mid \mathcal{G})+\mathrm{E}(\gamma \mid \mathcal{G}), \\
\mathrm{E}(c \gamma \mid \mathcal{G}) & =c \mathrm{E}(\gamma \mid \mathcal{G})
\end{aligned}
$$

for every $\beta, \gamma \in \mathrm{L}_{1}(\Omega, \mathcal{F}, \mathrm{P})$ and $c \in \mathbb{C}$. Equality holds for $\overline{\mathbb{R}}_{+}$-valued $\beta$ and $\gamma$, as well, which is immediate from the definition of extended conditional expectation. In particular,

$$
\mathrm{E}(|\gamma| \mid \mathcal{G})=\mathrm{E}\left(\gamma_{+} \mid \mathcal{G}\right)+\mathrm{E}\left(\gamma_{-} \mid \mathcal{G}\right)
$$

for every $\mathbb{R}$-valued random variable $\gamma$.

The next two statements are immediate from the definition of extended conditional expectation.

Lemma 2.1. Let $\gamma$ be an $\mathbb{R}$-valued random variable such that $\mathrm{E}(\gamma \mid \mathcal{G})$ exists. Then Equality (3) holds for every $c \in \mathbb{R}$.

Lemma 2.2. Let $\Xi$ be an $\overline{\mathbb{R}}_{+}$-valued random variable. Then for any $S \in \mathcal{G} \mathrm{E}\left(\Xi I_{S} \mid \mathcal{G}\right)=\mathrm{E}(\Xi \mid \mathcal{G}) I_{S}$.

Lemma 2.3. Let $\beta$ and $\gamma$ be nonnegative random variables such that $\beta \leq \gamma$. Then $\mathrm{E}(\beta \mid \mathcal{G}) \leq \mathrm{E}(\gamma \mid \mathcal{G})$.

Proof. Denote

$\chi=I\{\mathrm{E}(\gamma \mid \mathcal{G})<\infty\}, \Gamma=(\mathrm{E}(\gamma \mid \mathcal{G})-\mathrm{E}(\beta \mid \mathcal{G})) \chi$. The assumption $\beta \leq \gamma$ and the definition of extended conditional expectation yield $\mathrm{E} \Gamma I_{G} \geq 0$ for every $G \in \mathcal{G}$. Consequently $\Gamma I\{\Gamma<0\}=0$.

Lemma 2.4. Let $\Xi$ be an $\overline{\mathbb{R}}_{+}$-valued random variable. Then for any $\varepsilon>0$ and $a>0$

$$
\mathrm{P}\{\Xi>\varepsilon\} \leq a / \varepsilon+\mathrm{P}\left\{\mathrm{E}^{0} \Xi>a\right\} .
$$

Proof. By Formula (1)

$\mathrm{P}\left\{\Xi>\varepsilon, \mathrm{E}^{0} \Xi \leq a\right\}=\operatorname{EE}^{0} I\left\{\Xi>\varepsilon, \mathrm{E}^{0} \Xi \leq a\right\}$. By Lemma $2.2 \mathrm{E}^{0} I\left\{\Xi>\varepsilon, \mathrm{E}^{0} \Xi \leq a\right\}=I\left\{\mathrm{E}^{0} \Xi \leq a\right\} \mathrm{P}^{0}\{\Xi>\varepsilon\}$. Вy Lemmas 2.3 and $2.1 \mathrm{E}^{0} \Xi \geq \varepsilon \mathrm{P}^{0}\{\Xi>\varepsilon\}$ and therefore $I\left\{\mathrm{E}^{0} \Xi \leq a\right\} \mathrm{P}^{0}\{\Xi>\varepsilon\} \leq a / \varepsilon$. It remains to write the evident inclusion

$$
\{\Xi>\varepsilon\} \subset\left\{\Xi>\varepsilon, \mathrm{E}^{0} \Xi \leq a\right\} \cup\left\{\mathrm{E}^{0} \Xi>a\right\} \text {. }
$$

Corollary 2.5. Let $\mathcal{F}^{0} \subset \mathcal{G} \subset \mathcal{F}$ and let $\gamma$ be a nonnegative random variable such that $\mathrm{E}^{0} \gamma<\infty$. Then $\mathrm{E}(\gamma \mid \mathcal{G})<\infty$.

Proof. Lemma 2.4 and Formula (1) yield for arbitrary $N>0$ and $a>0$

$$
\mathrm{P}\{\mathrm{E}(\gamma \mid \mathcal{G})>N\} \leq a / N+\mathrm{P}\left\{\mathrm{E}^{0} \gamma>a\right\} .
$$

Passing in this inequality to the limit at first as $N \rightarrow \infty$ and hereafter as $a \rightarrow \infty$, we get

$$
\lim _{N \rightarrow \infty} \mathrm{P}\{\mathrm{E}(\gamma \mid \mathcal{G})>N\}=0
$$

Lemma 2.6. Let $\left(\beta_{n}\right)$ be an increasing sequence of $\overline{\mathbb{R}}_{+}$-valued random variables. Then $\mathrm{E} \lim \beta_{n}=\lim \mathrm{E} \beta_{n}$.

Proof. In case the r.h.s is finite this is the Beppo Levi theorem. Having written $\mathrm{E} \lim \beta_{m} \geq \mathrm{E} \beta_{n}$, we obtain the same equality when $\mathrm{E} \beta_{n} \rightarrow \infty . \square$

Lemma 2.7. Let $\left(\Gamma_{n}\right)$ be an increasing sequence of $\overline{\mathbb{R}}_{+}$-valued random variables. Then

$$
\mathrm{E}\left(\lim \Gamma_{n} \mid \mathcal{G}\right)=\lim \mathrm{E}\left(\Gamma_{n} \mid \mathcal{G}\right) .
$$


Proof. Denote $\Gamma=\lim \Gamma_{n}, \phi_{n}=\mathrm{E}\left(\Gamma_{n} \mid \mathcal{G}\right), \phi=\lim \phi_{n}$. By construction $\phi$ is $\mathcal{G}$-measurable. Lemma 2.6 and the definition of conditional expectation yield, for arbitrary $G \in \mathcal{G}$,

$\mathrm{E} \phi I_{G}=\lim \mathrm{E} \phi_{n} I_{G}, \mathrm{E} \phi_{n} I_{G}=\mathrm{E} \Gamma_{n} I_{G}, \lim \mathrm{E} \Gamma_{n} I_{G}=\mathrm{E} \Gamma I_{G}$.

So $\mathrm{E} \phi I_{G}=\mathrm{E} \Gamma I_{G}$, which in view of $\mathcal{G}$-measurability of $\phi$ proves the lemma. $\square$

Corollary 2.8. For every sequence $\left(\gamma_{n}\right)$ of nonnegative random variables the inequality

$\mathrm{E}\left(\underline{\lim }_{n} \mid \mathcal{G}\right) \leq \underline{\operatorname{limE}}\left(\gamma_{n} \mid \mathcal{G}\right)$ is valid.

Proof. Denote $\Gamma_{n}=\inf _{k \geq n} \gamma_{k}, \Gamma=\underline{\lim \gamma_{n}}$. By Lemma 2.3 $\mathrm{E}\left(\Gamma_{n} \mid \mathcal{G}\right) \leq \inf _{k \geq n} \mathrm{E}\left(\gamma_{k} \mid \mathcal{G}\right)$. Herein $\Gamma_{n} \nearrow \Gamma$, whence by Lemma $2.7 \mathrm{E}\left(\Gamma_{n} \mid \mathcal{G}\right) \rightarrow \mathrm{E}(\Gamma \mid \mathcal{G})$.

Lemma 2.9. Let $\left(\Gamma_{n}\right)$ be a decreasing sequence of nonnegative random variables such that $\mathrm{E}\left(\Gamma_{1} \mid \mathcal{G}\right)<\infty$. Then $\mathrm{E}\left(\lim \Gamma_{n} \mid \mathcal{G}\right)=\lim \mathrm{E}\left(\Gamma_{n} \mid \mathcal{G}\right)$.

Proof. Retaining the notation of the proof of Lemma 2.7, we denote additionally

$\Lambda=\mathrm{E}\left(\Gamma_{1} \mid \mathcal{G}\right), A^{N}=\{\Lambda \leq N\}(\in \mathcal{G})$. Then from the definition of conditional expectation we have

$$
\mathrm{E} \phi_{n} I_{A^{N}} I_{G}=\mathrm{E} \Gamma_{n} I_{A^{N}} I_{G}
$$

for arbitrary $N>0$ and $G \in \mathcal{G}$. By condition $\phi_{n} \leq \Lambda$, so $0 \leq \mathrm{E} \phi_{n} I_{A^{N}} I_{G} \leq N, 0 \leq \mathrm{E} \Gamma_{n} I_{A^{N}} I_{G} \leq N$, whence, taking to account monotonicity of $\left(\Gamma_{n}\right)$ (and therefore of $\left(\phi_{n}\right)$ ) we conclude by the Beppo Levi theorem that $\mathrm{E} \Gamma I_{A^{N}} I_{G}=\lim \mathrm{E} \Gamma_{n} I_{A^{N}} I_{G}, \mathrm{E} \phi I_{A^{N}} I_{G}=\lim \mathrm{E} \phi_{n} I_{A^{N}} I_{G}$. Juxtaposing these two equalities with (5), we see that

$$
\mathrm{E} \phi I_{G} I_{A^{N}}=\mathrm{E} \Gamma I_{G} I_{A^{N}}
$$

for any $N>0$. Herein $I_{A^{N}} \rightarrow 1$ as $N \rightarrow \infty$, since by assumption $\mathrm{P}\{\Lambda<\infty\}=1$. Then from (6) we get by Lemma 2.6 $\mathrm{E} \phi I_{G}=\mathrm{E} \Gamma I_{G}$. $\square$

Theorem 2.10. Let $\left(\rho_{n}\right)$ be a sequence of $\mathbb{R}^{d}$ valued random variables almost surely converging to a random variable $\rho$ and such that

$$
\mathrm{E}\left(\sup _{n}\left|\rho_{n}\right| \mid \mathcal{G}\right)<\infty .
$$

Then $\mathrm{E}(|\rho| \mid \mathcal{G})<\infty$ and $\mathrm{E}\left(\rho_{n} \mid \mathcal{G}\right) \rightarrow \mathrm{E}(\rho \mid \mathcal{G})$.

Proof. Let first the $\rho_{n}$ 's be nonnegative. Denote $\gamma_{n}=\inf _{k \geq n} \rho_{k}, \Gamma_{n}=\sup _{k \geq n} \rho_{k}$. Then

$$
\gamma_{n} \leq \rho_{n} \leq \Gamma_{n},
$$

$\gamma_{n} \nearrow \rho, \Gamma_{n} \searrow \rho$. From the second relation we have by Corollary $2.8 \mathrm{E}(\rho \mid \mathcal{G}) \leq \underline{\operatorname{limE}}\left(\gamma_{n} \mid \mathcal{G}\right)$; the third relation together with (7) yields by Lemma 2.9

$\mathrm{E}(\rho \mid \mathcal{G})=\lim \mathrm{E}\left(\Gamma_{n} \mid \mathcal{G}\right)$. Comparing these two conclusions with (8), we get $\mathrm{E}(\rho \mid \mathcal{G})=\lim \mathrm{E}\left(\rho_{n} \mid \mathcal{G}\right)$. Thus we have proved the theorem for nonnegative random variables. The transition to the general case is trivial.

Lemma 2.11. Let $\beta$ and $\gamma$ be $\mathbb{R}^{d}$-valued random variables such that the conditional expectations $\mathrm{E}(\beta \mid \mathcal{G})$ and $\mathrm{E}(\gamma \mid \mathcal{G})$ exist and are component-wise finite. Then $\mathrm{E}(\beta+\gamma \mid \mathcal{G})$ exists and Equality (2) holds.

Proof. The assumptions of the lemma together with Equality (4) imply that

$$
\mathrm{E}(|\beta| \mid \mathcal{G})<\infty, \quad \mathrm{E}(|\gamma| \mid \mathcal{G})<\infty .
$$

For nonnegative random variables Equality (2) ensues, as was pointed out above, directly from the definition of extended conditional expectation, so Inequalities (9) yield

$$
\mathrm{E}(|\beta|+|\gamma| \mid \mathcal{G})<\infty
$$

Denote, for each $n \in \mathbb{N}$,

$$
\beta_{n}=\frac{n \beta}{n \vee|\beta|}, \quad \gamma_{n}=\frac{n \gamma}{n \vee|\gamma|},
$$

$\rho_{n}=\beta_{n}+\gamma_{n}$. By construction $\left|\beta_{n}\right| \leq n,\left|\gamma_{n}\right| \leq n$ and therefore $\beta_{n}, \gamma_{n} \in \mathrm{L}_{1}(\Omega, \mathcal{F}, \mathrm{P})$. Consequently,

$\mathrm{E}\left(\rho_{n} \mid \mathcal{G}\right)=\mathrm{E}\left(\beta_{n} \mid \mathcal{G}\right)+\mathrm{E}\left(\gamma_{n} \mid \mathcal{G}\right)$.

Obviously, $\beta_{n} \rightarrow \beta, \gamma_{n} \rightarrow \gamma, \rho_{n} \rightarrow \beta+\gamma$. Herein by construction $\left|\beta_{n}\right| \leq|\beta|,\left|\gamma_{n}\right| \leq|\gamma|,\left|\rho_{n}\right| \leq|\beta|+|\gamma|$, which together with (10) and (9) implies (7) and the same for $\left(\beta_{n}\right)$ and $\left(\gamma_{n}\right)$. Hence and from the above asymptotic relations we get by Theorem 2.10

$$
\begin{aligned}
& \mathrm{E}\left(\beta_{n} \mid \mathcal{G}\right) \rightarrow \mathrm{E}(\beta \mid \mathcal{G}), \mathrm{E}\left(\gamma_{n} \mid \mathcal{G}\right) \rightarrow \mathrm{E}(\gamma \mid \mathcal{G}), \\
& \mathrm{E}\left(\rho_{n} \mid \mathcal{G}\right) \rightarrow \mathrm{E}(\beta+\gamma \mid \mathcal{G}) .
\end{aligned}
$$

Lemma 2.12. Let $\mathcal{F}^{0} \subset \mathcal{G} \subset \mathcal{F}$ and $\gamma$ be a $\mathbb{R}^{d}$ valued random variable such that $\mathrm{E}^{0}|\gamma|<\infty$. Then $\mathrm{E}^{0} \gamma=\mathrm{E}^{0} \mathrm{E}(\gamma \mid \mathcal{G})$.

Proof. It suffices to consider the case $d=1$. Then the last assumption of the lemma amounts to $\mathrm{E}^{0} \gamma_{ \pm}<\infty$. Denote $\Gamma_{1}=\mathrm{E}\left(\gamma_{+} \mid \mathcal{G}\right), \Gamma_{2}=\mathrm{E}\left(\gamma_{-} \mid \mathcal{G}\right)$. By Formula (1) $\mathrm{E}^{0} \Gamma_{1,2}=\mathrm{E}^{0} \gamma_{ \pm}$and therefore $\mathrm{E}^{0} \Gamma_{1,2}<\infty$. Then by Lemma $2.11 \mathrm{E}^{0}\left(\Gamma_{1}-\Gamma_{2}\right)=\mathrm{E}^{0} \Gamma_{1}-\mathrm{E}^{0} \Gamma_{2}$, which together with the previous inequality and the definition of extended conditional expectation yields

$\mathrm{E}^{0}\left(\Gamma_{1}-\Gamma_{2}\right)=\mathrm{E}^{0} \gamma$. The inequalities $\mathrm{E}^{0} \Gamma_{1,2}<\infty$ imply, by Corollary 2.5 , that $\Gamma_{1,2}<\infty$, whence by the definitions of $\Gamma_{i}$ and extended conditional expectation we have $\Gamma_{1}-\Gamma_{2}=\mathrm{E}(\gamma \mid \mathcal{G})$. $\square$

Lemma 2.13. Let $\Upsilon$ and $\Xi$ be nonnegative random variables, $\Upsilon$ be $\mathcal{G}$-measurable. Then $\mathrm{E}(\Upsilon \Xi \mid \mathcal{G})=\Upsilon \mathrm{E}(\Xi \mid \mathcal{G})$.

Proof. Denote $S_{n k}=\left\{k 2^{-n}<\Upsilon \leq(k+1) 2^{-n}\right\} \quad(\in \mathcal{G}$ due to $\mathcal{G}$-measurability of $\Upsilon), \quad J_{n k}=I_{S_{n k}}$,

$$
\Upsilon_{n m}=2^{-n} \sum_{k=1}^{m} k J_{n k}, \Upsilon_{n}=2^{-n} \sum_{k=1}^{\infty} k J_{n k} \text {. }
$$

Formula (2) (for nonnegative random variables), Lemma 2.1 and the definition of $\Upsilon_{n m}$ yield 
$\mathrm{E}\left(\Upsilon_{n m} \Xi \mid \mathcal{G}\right)=2^{-n} \sum_{k=1}^{m} k \mathrm{E}\left(J_{n k} \Xi \mid \mathcal{G}\right)$. Noting that

$\mathrm{E}\left(J_{n k} \Xi \mid \mathcal{G}\right)=\mathrm{E}(\Xi \mid \mathcal{G}) J_{n k}$ by Lemma 2.2, we convert this equality to $\mathrm{E}\left(\Upsilon_{n m} \Xi \mid \mathcal{G}\right)=\Upsilon_{n m} \mathrm{E}(\Xi \mid \mathcal{G})$. Obviously, $\Upsilon_{n m} \nearrow \Upsilon_{n}$ as $m \rightarrow \infty$. Then by Lemma 2.7

$\mathrm{E}\left(\Upsilon_{n m} \mid \mathcal{G}\right) \nearrow \mathrm{E}\left(\Upsilon_{n} \mid \mathcal{G}\right)$ as $m \rightarrow \infty$, which together with the last equality yields $\mathrm{E}\left(\Upsilon_{n} \Xi \mid \mathcal{G}\right)=\Upsilon_{n} \mathrm{E}(\Xi \mid \mathcal{G})$. It remains to let $n \rightarrow \infty$ and again make use of Lemma 2.7. $\square$

Lemma 2.14. Let $\Upsilon$ and $\Xi$ be random variables with values in $\mathbb{R}^{d}$ and $\mathbb{R}^{p}$, respectively. Suppose that $\Upsilon$ is $\mathcal{G}$-measurable and $\mathrm{E}(|\Xi| \mid \mathcal{G})<\infty$. Then $\mathrm{E}\left(\Upsilon \Xi^{\mathrm{T}} \mid \mathcal{G}\right)=\Upsilon \mathrm{E}\left(\Xi^{\mathrm{T}} \mid \mathcal{G}\right)$.

Proof. It suffices to consider the case $d=1, p=1$. Writing, for arbitrary $a, b \in \mathbb{R}$, the evident equalities $(a b)_{+}=a_{+} b_{+}+a_{-} b_{-},(a b)_{-}=a_{+} b_{-}+a_{-} b_{+}$, we get from Lemma 2.13

$$
\begin{aligned}
& \mathrm{E}\left((\Upsilon \Xi)_{+} \mid \mathcal{G}\right)=\Upsilon_{+} \mathrm{E}\left(\Xi_{+} \mid \mathcal{G}\right)+\Upsilon_{-} \mathrm{E}\left(\Xi_{-} \mid \mathcal{G}\right), \\
& \mathrm{E}\left((\Upsilon \Xi)_{-} \mid \mathcal{G}\right)=\Upsilon_{+} \mathrm{E}\left(\Xi_{-} \mid \mathcal{G}\right)+\Upsilon_{-} \mathrm{E}\left(\Xi_{+} \mid \mathcal{G}\right) .
\end{aligned}
$$

The assumption $\mathrm{E}(|\Xi| \mid \mathcal{G})<\infty$ implies finiteness of the right-hand sides of both equalities. Consequently, the left-hand sides are finite, too. Then by the definition of extended conditional expectation

$\mathrm{E}(\Upsilon \Xi \mid \mathcal{G})=\mathrm{E}\left((\Upsilon \Xi)_{+} \mid \mathcal{G}\right)-\mathrm{E}\left((\Upsilon \Xi)_{-} \mid \mathcal{G}\right)$, which together with the two preceding equalities completes the proof. $\square$

Lemma 2.15. Let $\mathcal{F}^{0} \subset \mathcal{G} \subset \mathcal{F}$, and let $\Upsilon$ and $\Xi$ be random variables with values in $\mathbb{R}^{d}$ and $\mathbb{R}^{p}$, respectively, such that:

$\mathrm{E}^{0}|\mathrm{\Upsilon}||\Xi|<\infty, \mathrm{E}(|\Xi| \mid \mathcal{G})<\infty, \mathrm{E}(\Xi \mid \mathcal{G})=0$ and $\Upsilon$ is $\mathcal{G}$ measurable. Then $\mathrm{E}^{0} \Upsilon \Xi^{\mathrm{T}}=O$ (the null matrix).

Proof. From the last three assumptions we get by Lemma $2.14 \mathrm{E}(\Upsilon \Xi \mid \mathcal{G})=O$; the first assumption implies, according to Lemma 2.12, the equality

$\mathrm{E}^{0} \Upsilon \Xi^{\mathrm{T}}=\mathrm{E}^{0} \mathrm{E}\left(\Upsilon \Xi^{\mathrm{T}} \mid \mathcal{G}\right)$.

Lemma 2.16. Let $\left(\Xi_{n}\right)$ be a converging in probability to zero sequence of nonnegative random variables such that for some increasing unbounded function $F: \mathbb{R}_{+} \rightarrow \mathbb{R}_{+}$the sequence $\left(\mathrm{E}\left(\Xi_{n} F\left(\Xi_{n}\right) \mid \mathcal{G}\right)\right)$ is stochastically bounded. Then $\mathrm{E}\left(\Xi_{n} \mid \mathcal{G}\right) \stackrel{\mathrm{P}}{\longrightarrow} 0$.

Proof. From the first assumption we have $\mathrm{E}\left(\Xi_{n} I\left\{\Xi_{n} \leq N\right\} \mid \mathcal{G}\right) \stackrel{\mathrm{P}}{\longrightarrow} 0$ for every $N>0$, so it suffices to show that for any $\varepsilon>0$

$$
\lim _{N \rightarrow \infty} \varlimsup_{n \rightarrow \infty} \mathrm{P}\left\{\mathrm{E}\left(\Xi_{n} I\left\{\Xi_{n}>N\right\} \mid \mathcal{G}\right)>\varepsilon\right\}=0 .
$$

Since $F$ increases to infinity, we shall have $\Xi_{n} I\left\{\Xi_{n}>N\right\} \leq F(N)^{-1} \Xi_{n} F\left(\Xi_{n}\right)$ for sufficiently large $N$ (such that $F(N)>0$ ). Then by Lemma 2.3

$$
\begin{aligned}
& \varlimsup_{n \rightarrow \infty} \mathrm{P}\left\{\mathrm{E}\left(\Xi_{n} I\left\{\Xi_{n}>N\right\} \mid \mathcal{G}\right)>\varepsilon\right\} \\
& \leq \varlimsup_{n \rightarrow \infty} \mathrm{P}\left\{\mathrm{E}\left(\Xi_{n} F\left(\Xi_{n}\right) \mid \mathcal{G}\right)>\varepsilon F(N)\right\}
\end{aligned}
$$

for those $N$. Letting here $N \rightarrow \infty$, we deduce (11) from the last assumption of the lemma and unbounded growth of $F$. $\square$

Lemma 2.17. Let $\varphi$ be an $\mathbb{R}_{+}$-valued measurable random process. Then for any $\mathcal{F}^{0}$-measurable random variable $\tau$ we have

$$
\mathrm{E}^{0} \varphi(\tau)=\left.\mathrm{E}^{0} \varphi(s)\right|_{s=\tau} .
$$

Proof. Denote

$$
\begin{aligned}
& \mathcal{C}=\left\{C \in \mathcal{F} \otimes \mathcal{B}_{+}: \mathrm{E}^{0} I_{C}(\cdot, \tau(\cdot))=\left.\mathrm{E}^{0} I_{C}(\cdot, s)\right|_{s=\tau(\cdot)}\right\} . \text { Let } \\
& Q \in \mathcal{F}, B \in \mathcal{B}_{+} \text {. Then } I_{Q \times B}(\omega, \tau(\omega))=I_{Q}(\omega) I_{\tau^{-1}(B)}(\omega),
\end{aligned}
$$

whence by the assumption about $\tau$ and by Lemma 2.2 we have

$$
\mathrm{E}^{0} I_{Q \times B}(\tau)=\left.I_{\tau^{-1}(B)} \mathrm{E}^{0} I_{Q} \equiv \mathrm{E}^{0}\left(I_{Q} I_{B}(s)\right)\right|_{s=\tau} .
$$

Thus $\mathcal{C}$ contains all sets of the kind $Q \times B$, where $Q \in \mathcal{F}, B \in \mathcal{B}_{+} \quad$ ("measurable rectangles"). Then it follows from (2) (for nonnegative random variables) that $\mathcal{C}$ contains also all possible finite unions of pairwise disjoint measurable rectangles. According to Lemma 2.6 $\mathcal{C}$ contains the union of every increasing sequence of its members. Consequently, it contains the $\sigma$-algebra generated by measurable rectangles, i.e. Equality (12) holds for $\varphi=I_{C}, C \in \mathcal{F} \otimes \mathcal{B}_{+}$.

Passing to the general case, we denote $C_{n k}=\left\{k 2^{-n}<\varphi \leq(k+1) 2^{-n}\right\} \quad\left(\in \mathcal{F} \otimes \mathcal{B}_{+}\right.$due to measurability of $\varphi), \chi_{n k}=I_{C_{n k}}, \varphi_{n}=2^{-n} \sum_{k=1}^{\infty} k \chi_{n k}$. By construction $\varphi_{n}(s) \nearrow \varphi(s)$ for all $\omega$ and $s$ and therefore $\varphi_{n}(\tau) \nearrow \varphi(\tau)$. From these relations we get by Lemma 2.7

$$
\mathrm{E}^{0} \varphi_{n}(s) \nearrow \mathrm{E}^{0} \varphi(s), \quad \mathrm{E}^{0} \varphi_{n}(\tau) \nearrow \mathrm{E}^{0} \varphi(\tau)
$$

As was shown (in another notation) in the proof of Lemma 2.13, $\mathrm{E}^{0} \varphi_{n}(\tau)=2^{-n} \sum_{k} k \mathrm{E}^{0} \chi_{n k}(\tau)$. By what was proved $\mathrm{E}^{0} \chi_{n k}(\tau)=\left.\mathrm{E}^{0} \chi_{n k}(s)\right|_{s=\tau}$, which together with the previous equality yields

$\mathrm{E}^{0} \varphi_{n}(\tau)=\left.\mathrm{E}^{0} \varphi_{n}(s)\right|_{s=\tau}$. Juxtaposing this with (13), we arrive at (12). $\square$

In the next two statements, the process $\varphi$ need not be càdlàg.

Lemma 2.18. Let $H \in \mathcal{V}_{0}^{+}$and $\varphi$ be a bounded measurable random process on $[a, b] \subset \mathbb{R}_{+}$. Then

$$
\mathrm{E}^{0} \int_{a}^{b} \varphi(s) \mathrm{d} H(s)=\int_{a}^{b} \mathrm{E}^{0} \varphi(s) \mathrm{d} H(s) .
$$

Proof. 1) Lemma 2.11 allows to consider, without loss of generality, that $\phi$ is $\mathbb{R}_{+}$-valued. Then the boundedness assumption together with Lemma 2.1 allows to consider that $0 \leq \varphi \leq 1$.

Let at first $\varphi(s)=\gamma f(s)$, where $\gamma$ and $f$ are a random variable and a Borel function, respectively. Then 
Equality (14) follows from Lemma 2.13.

2) Let for all $s \in[a, b] \varphi_{n}(s) \nearrow \varphi(s)$, where $\left(\varphi_{n}\right)$ is an increasing sequence of $[0,1]$-valued random processes such that for each $n$

$$
\mathrm{E}^{0} \int_{a}^{b} \varphi_{n}(s) \mathrm{d} H(s)=\int_{a}^{b} \mathrm{E}^{0} \varphi_{n}(s) \mathrm{d} H(s) .
$$

Then: for any $s$ the sequence $\left(\mathrm{E}^{0} \varphi_{n}(s)\right)$ increases by Lemma 2.3 and $\mathrm{E}^{0} \varphi_{n}(s) \rightarrow \mathrm{E}^{0} \varphi(s)$ by Lemma 2.13; $\int_{a}^{b} \varphi_{n}(s) \mathrm{d} H(s) \nearrow \int_{a}^{b} \varphi(s) \mathrm{d} H(s)$ by the Beppo Levi theorem. By the same theorem we get from the first relation $\int_{a}^{b} \mathrm{E}^{0} \varphi_{n}(s) \mathrm{d} H(s) \rightarrow \int_{a}^{b} \mathrm{E}^{0} \varphi(s) \mathrm{d} H(s)$. The second relation jointly with Lemma 2.13 yields

$\mathrm{E}^{0} \int_{a}^{b} \varphi_{n}(s) \mathrm{d} H(s) \rightarrow \mathrm{E}^{0} \int_{a}^{b} \varphi(s) \mathrm{d} H(s)$. Comparing these two conclusions with (15), we obtain (14).

3) Let $\mathcal{C}$ denote the class of all

$C \in \mathcal{F} \otimes\left(\mathcal{B}_{+} \cap 2^{[a, b]}\right)$ such that Equality (14) holds for $\varphi=I_{C}$. According to item 1) $\mathcal{C}$ contains the algebra generated by measurable triangles. Then it follows from item 2) that $\mathcal{C} \supset \mathcal{F} \otimes\left(\mathcal{B}_{+} \cap 2^{[a, b]}\right)$.

4) Let us define the sequence $\left(\varphi_{n}\right)$ by

$\varphi_{n}=2^{-n} \sum_{k=0}^{2^{n}-1} k \chi_{n k}$, where the $\chi_{n k}$ 's are the same as in the proof of Lemma 2.17. Item 3), Lemma 2.11 and Lemma 2.1 imply together (15) for each $n$. Herein by construction $\varphi_{n} \nearrow \varphi$. It remains to refer to item 2). $\square$

Theorem 2.19. Let $H \in \mathcal{V}_{0}^{+}$and $\varphi$ be a nonnegative measurable random process on $[a, b] \subset \mathbb{R}_{+}$. Then Equality (14) holds with possible value $\infty$ of both sides. Proof. By Lemma 2.18 for any $n \in \mathbb{N}$

$$
\mathrm{E}^{0} \int_{a}^{b}(\varphi(s) \wedge n) \mathrm{d} H(s)=\int_{a}^{b} \mathrm{E}^{0}(\varphi(s) \wedge n) \mathrm{d} H(s) .
$$

By Lemma 2.6 for any $s$

$$
\mathrm{E}^{0}(\varphi(s) \wedge n) \rightarrow \mathrm{E}^{0} \varphi(s) .
$$

By the same argument as in the proof of that lemma,

$$
\int_{a}^{b}(\varphi(s) \wedge n) \mathrm{d} H(s) \rightarrow \int_{a}^{b} \varphi(s) \mathrm{d} H(s)
$$

and, in view of (17),

$$
\int_{a}^{b} \mathrm{E}^{0}(\varphi(s) \wedge n) \mathrm{d} H(s) \rightarrow \int_{a}^{b} \mathrm{E}^{0} \varphi(s) \mathrm{d} H(s)
$$

From (18) we have by Lemma 2.6

$\mathrm{E}^{0} \int_{a}^{b}(\varphi(s) \wedge n) \mathrm{d} H(s) \rightarrow \int_{a}^{b} \mathrm{E}^{0} \varphi(s) \mathrm{d} H(s)$ which together with (16) and (19) proves (14).

\section{A Subclass of the Class of Locally Square Integrable Martingales}

The stochastic integral $\int_{0}^{t} \zeta(s) \mathrm{d} X(s)$ w.r.t. a local mar- tingale $X$ will be written, following [5,6], as $\zeta \cdot X(t)$. The designation of this section is to find the least restrictive extra assumptions providing the properties

$$
\begin{aligned}
& \mathrm{E}^{0}|\zeta \cdot X(t)|^{2}<\infty, \\
& \mathrm{E}(\zeta \cdot X(t \vee s) \mid \mathcal{F}(t \wedge s))=\zeta \cdot X(t \wedge s)
\end{aligned}
$$

of $\zeta \cdot X$ underlying the derivations in Section 4. Herein we do not demand that $\mathrm{E}|\zeta \cdot X(t)|<\infty$, so the conditional expectations in these properties are not classical but extended.

The following statement differs from Doob's optional theorem for nonnegative discrete-time submartingales only with the absence of the demand $\mathrm{E} \gamma_{k}<\infty$ falling out of the proof if one uses the extended expectation instead of the ordinary one.

Lemma 3.1. Let $\left(\gamma_{k}, k \in \mathbb{Z}_{+}\right)$be a sequence of nonnegative random variables adapted to a flow

$\left(\mathcal{G}_{k}, k \in \mathbb{Z}_{+}\right)$and such that $\mathrm{E}\left(\gamma_{k} \mid \mathcal{G}_{k-1}\right) \geq \gamma_{k-1}, k \in \mathbb{N}$.

Then the inequality $\mathrm{E}\left(\gamma_{\tau} \mid \mathcal{G}_{\sigma}\right) \geq \gamma_{\sigma}$ holds for any bounded stopping times (w.r.t. the same flow) $\tau$ and $\sigma \leq \tau$.

This result leads in the standard way to Doob's inequality asserted by the following lemma.

Lemma 3.2. Under the assumptions of Lemma 3.1, $\mathrm{E}\left(\max _{k \leq n} \gamma_{k}^{2} \mid \mathcal{G}_{0}\right) \leq 4 \mathrm{E}\left(\gamma_{n}^{2} \mid \mathcal{G}_{0}\right), n \in \mathbb{N}$.

Let $\underline{\mathcal{K}}$ denote the class of all $\mathbb{F}$-adapted $\mathbb{R}^{d}$ valued ( $d$ will be determined by the context, if matters) random processes $M$ satisfying the conditions:

$$
\begin{aligned}
& \text { M1. For all } t \quad \mathrm{E}^{0}|M(t)|^{2}<\infty \text {. } \\
& \text { M2. For all } t \geq s \geq 0 \quad \mathrm{E}(M(t) \mid \mathcal{F}(s))=M(s) \text {. }
\end{aligned}
$$

Lemma 3.3. Let $M \in \underline{\mathcal{K}}$. Then $\mathrm{E}^{0}(M(t)-M(s)) \Upsilon^{\mathrm{T}}=\bar{O}$ for every $t>s \geq 0, p \in \mathbb{N}$ and $\mathcal{F}(s)$-measurable $\mathbb{R}^{p}$-valued random variable $\Upsilon$ such that $\mathrm{E}^{0}|M(t)-M(s)||\Upsilon|<\infty$.

Proof. Denote $\Xi=M(t)-M(s)$. Then:

$\mathrm{E}(\Xi \mid \mathcal{F}(s))=0$ by condition M2 and the assumption that $M$ is $\mathbb{F}$-adapted; $\mathrm{E}^{0}(\mid \Xi \| \mathcal{F}(s))<\infty$ by condition M1. It remains to refer to Lemma 2.15.

Corollary 3.4. (from Lemmas 3.3 and 2.3) Let $M \in \underline{\mathcal{K}}$. Then for all $t>s \geq 0$

$$
\mathrm{E}^{0}(M(t)-M(s)) M(s)^{\mathrm{T}}=O .
$$

Hence and from the identity $|x|^{2}=\operatorname{tr} x x^{\mathrm{T}} \quad\left(x \in \mathbb{R}^{d}\right)$ we get

Corollary 3.5. Let $M \in \underline{\mathcal{K}}$. Then for all $t>s \geq 0 \quad \mathrm{E}^{0}|M(t)-M(s)|^{2}=\mathrm{E}^{0}|M(t)|^{2}-\mathrm{E}^{0}|M(s)|^{2}$.

Lemma 3.6. Let $M \in \underline{\mathcal{K}}$. Then for any $t_{1}>t_{0} \geq 0$

$$
\mathrm{E}\left(\sup _{t_{0} \leq t \leq t_{1}}|M(t)|^{2} \mid \mathcal{F}\left(t_{0}\right)\right) \leq 4 \mathrm{E}\left(|M(t)|^{2} \mid \mathcal{F}\left(t_{0}\right)\right) .
$$


Proof. Denote

$t_{n k}=t_{0}+k 2^{-n}\left(t_{1}-t_{0}\right), \gamma_{n k}=\left|M\left(t_{n k}\right)\right|, \Gamma_{n}=\max _{0 \leq k \leq 2^{n}} \gamma_{n k}^{2}$. By

construction and condition M1

$\mathrm{E}\left(\gamma_{n k} \mid \mathcal{F}\left(t_{n k-1}\right)\right) \geq\left|\mathrm{E}\left(M\left(t_{n k}\right) \mid \mathcal{F}\left(t_{n k-1}\right)\right)\right|=\gamma_{n k-1}$, whence by Lemma 3.2

$\mathrm{E}\left(\Gamma_{n} \mid \mathcal{F}\left(t_{0}\right)\right) \leq 4 \mathrm{E}\left(\gamma_{n 2^{n}} \mid \mathcal{F}\left(t_{0}\right)\right) \equiv 4 \mathrm{E}\left(\left|M\left(t_{1}\right)\right|^{2} \mid \mathcal{F}\left(t_{0}\right)\right)$.

Herein $M$ is càdlàg (see the first sentence of the article), so $\Gamma_{n} \nearrow \sup _{t_{0} \leq t \leq t_{1}}|M(t)|^{2}$. It remains to make use of Lemma 2.7. $\square$

Henceforth "stopping time" means "stopping time w.r.t. the flow $\mathbb{F}$ ".

Lemma 3.7. Let $M \in \underline{\mathcal{K}}$. Then the equality $\mathrm{E}(M(\sigma) \mid \mathcal{F}(s))=M(\sigma \wedge s)$ holds for every $s \in \mathbb{R}_{+}$ and bounded stopping time $\sigma$.

Proof. We consider, without loss of generality, $\mathbb{R}$-valued processes. Writing

$M(\sigma) I\{\sigma \leq s\}=M(\sigma \wedge s) I\{\sigma \leq s\}$ and noting that the r.h.s. of the equality is $\mathcal{F}(s)$-measurable, we get $\mathrm{E}(M(\sigma) I\{\sigma \leq s\} \mid \mathcal{F}(s))=M(\sigma \wedge s) I\{\sigma \leq s\}$. So it

suffices, in view of Lemma 2.11, to show that

$$
\begin{aligned}
& \mathrm{E}(M(\sigma) I\{\sigma>s\} \mid \mathcal{F}(s)) \\
& =M(s) I\{\sigma>s\}(\equiv M(\sigma \wedge s) I\{\sigma>s\}) .
\end{aligned}
$$

By assumption there exists a number $C$ such that $\sigma \leq C$. We will prove Equality (20) for $s<C$ (otherwise it is trivial). Denote

$N=2^{n}, s_{n 0}=s, s_{n k}=s+k 2^{-n}(C-s)$,

$I_{n k}=I\left\{s_{n k-1}<\sigma \leq s_{n k}\right\}, \quad \sigma_{n}=\sum_{k=1}^{N} s_{n k} I_{n k}$,

$\Xi_{n}=\left(M\left(\sigma_{n}\right)-M(\sigma)\right) I\{\sigma>s\}$. By construction $\sigma_{n}$ is a stopping time and $\sigma_{n} \searrow \sigma$ for all $\omega \in \Omega$. From the last relation and right-continuity of $M$ we have $\Xi_{n} \rightarrow 0$. Herein $\Xi_{n}^{2} \leq 4 \sup _{t \leq C} M(t)^{2}$, whence by Lemma $3.6 \mathrm{E}^{0} \Xi_{n}^{2} \leq 16 \mathrm{E}^{0} M(t)^{2}$, which in view of M1 proves stochastic boundedness of the sequence $\left(\Xi_{n}^{2}\right)$. Then by Lemma 2.16

$$
\mathrm{E}\left(\Xi_{n} \mid \mathcal{F}(s)\right) \stackrel{\mathrm{P}}{\longrightarrow} 0 .
$$

Denote $\mu_{n k}=M\left(s_{n k}\right), l_{n k}=I\left\{\sigma \leq s_{n k}\right\}, k=0, \cdots, N$.

From M1 we have by Corollary $2.5 \mathrm{E}\left(\left|\mu_{n k}\right| \mid \mathcal{F}(s)\right)<\infty$.

On the strength of $\mathbf{M} 2$

$\mathrm{E}\left(\mu_{n k+1}-\mu_{n k} \mid \mathcal{F}(s)\right)=M(s)-M(s)=0$, which together with the previous relation results, by Lemma 2.14, in

$$
\mathrm{E}\left(\left(\mu_{n k+1}-\mu_{n k}\right) \iota_{n k} I\{\sigma>s\} \mid \mathcal{F}(s)\right)=0 .
$$

By the same lemma and property $\mathbf{M} 2$ of $M$

$$
\mathrm{E}\left(\mu_{n N} I\{\sigma>s\} \mid \mathcal{F}(s)\right)=M(s) I\{\sigma>s\} .
$$

By the construction of $\sigma_{n}$

$$
\begin{aligned}
& M\left(\sigma_{n}\right)=\sum_{k=1}^{N} \mu_{n k} I_{n k} \\
& \equiv \mu_{n N} l_{n N}-\mu_{n 0} l_{n 0}-\sum_{k=1}^{N-1}\left(\mu_{n k+1}-\mu_{n k}\right) \iota_{n k} .
\end{aligned}
$$

Herein $\iota_{n 0} I\{\sigma>s\}=I\{s<\sigma \leq s\}=0$ and $u_{n N}=I\{\sigma \leq b\}=1$, which together with (24)-(22) and Lemma 2.11 yields

$\mathrm{E}\left(M\left(\sigma_{n}\right) I\{\sigma>s\} \mid \mathcal{F}(s)\right)=M(s) I\{\sigma>s\}$. This equality jointly with (21) proves (20).

The class of all random processes $M \in \underline{\mathcal{K}}$ such that the process $\mathrm{E}^{0}|M|^{2}$ is continuous will be denoted $\mathcal{K}$.

Lemma 3.8. $\mathcal{K}$ contains the sum of every two its elements.

Proof. Let $Z=X+Y$, where $X, Y \in \mathcal{K}$. Property M1 of $Z$ ensues from Lemma 2.11. It follows from Lemma 2.3 that $\mathrm{E}^{0}|Z(t)|^{2} \leq \mathrm{E}^{0}|X(t)|^{2}+\mathrm{E}^{0}|Y(t)|^{2}$, $\mathrm{E}^{0}|Z(t)-Z(s)|^{2} \leq \mathrm{E}^{0}|X(t)-X(s)|^{2}+\mathrm{E}^{0}|Y(t)-Y(s)|^{2}$ for all $t$ and $s$. Hence property $\mathbf{M} 2$ and, with account of Corollary 3.5, continuity of $\mathrm{E}^{0}|Z|^{2}$ emerge. $\square$

Theorem 3.9. $\mathcal{K} \subset \ell \mathcal{M}_{2}$.

Proof. Let $M \in \mathcal{K}$. Denote $U=\mathrm{E}^{0}|M|^{2}$, $\tau_{n}=\inf \{s: U(s) \geq n\}, \quad M_{n}(t)=M\left(t \wedge \tau_{n}\right)$. By construction all $\tau_{n}$ 's are $\mathcal{F}(0)$-measurable random variables (and therefore stopping times) and $\tau_{n} \nearrow \infty$. The process $|M|^{2}$ is $\mathbb{F}$-adapted and right-continuous and therefore, by Theorem 2.1.1 [1], $\mathbb{F}$-progressive and all the more measurable. Then Lemma 2.17 applied to $\varphi=|M|^{2}$ and $\sigma=t \wedge \tau_{n}$ yields $\mathrm{E}^{0}\left|M_{n}(t)\right|^{2}=U\left(t \wedge \tau_{n}\right)$. By Corollary $3.5 U$ is an increasing process and therefore $U\left(t \wedge \tau_{n}\right) \leq U\left(\tau_{n}\right)$. By the choice of $M$ the process $U$ is continuous, so $U\left(\tau_{n}\right) I\left\{\tau_{n}<\infty\right\}=n, U\left(\tau_{n}\right) \leq n$. Consequently, $\mathrm{E}^{0}\left|M_{n}(t)\right|^{2} \leq n$ and therefore $\mathrm{E}\left|M_{n}(t)\right|^{2} \leq n$. Herein by Lemma 3.7 $\mathrm{E}\left(M_{n}(t) \mid \mathcal{F}(s)\right)=M\left(t \wedge \tau_{n} \wedge s\right)$ $\left(\equiv M_{n}(s)\right.$ as $\left.t>s\right)$. Thus $\sup _{t} \mathrm{E}\left|M_{n}(t)\right|^{2}<\infty$ and $M_{n}$ is a martingale. This means, since $\left(\tau_{n}\right)$ is an increasing to infinity sequence of stopping times, that $M \in \ell \mathcal{M}_{2}$. $\square$

The quadratic variation of a semimartingale $S$ and the quadratic characteristic of a locally square integrable martingale $M$ will be denoted $[S]$ and $\langle M\rangle$, respectively.

The following statement is immediate from Theorem 1.8.1 in [5] and the definition of quadratic characteristic.

Lemma 3.10. Let $M$ be an $\mathbb{R}$-valued locally square integrable martingale. Then for any stopping time $\tau \quad \mathrm{E}^{0}(M(\tau)-M(0))^{2}=\mathrm{E}^{0}[M](\tau)=\mathrm{E}^{0}\langle M\rangle(\tau)$.

Corollary 3.11. Let $M$ be an $\mathbb{R}^{d}$-valued locally square integrable martingale. Then for any stopping time $\tau \quad \mathrm{E}^{0}(M(\tau)-M(0))^{2}=\mathrm{E}^{0} \operatorname{tr}[M](\tau)=\mathrm{E}^{0} \operatorname{tr}\langle M\rangle(\tau)$. 
Note that all the random variables $E^{0} \cdots$ in the above two statements are, generally speaking, $\overline{\mathbb{R}}_{+}$-valued.

The Lebesgue - Stieltjes integral $\int_{0}^{t} f(s) \mathrm{d} A(s)$, where $A$ is a random process of locally bounded variation, will be written shortly as $f \circ A(t)$.

In the next statement, the process $\mathfrak{z}$ need not be right-continuous and even may have second-kind discontinuities.

Lemma 3.12. Let $W$ be an $\mathbb{R}^{d}$-valued process of class $\mathcal{K}$ and $\mathfrak{z}$ be an $\mathbb{R}^{d *}$-valued $\mathbb{F}$-predictable random process such that

$$
\mathrm{E}^{0}\left(|\mathfrak{z}|^{2} \circ \operatorname{tr}\langle W\rangle(t)\right)<\infty, \quad t \in \mathbb{R}_{+},
$$

and the process $\mathrm{E}^{0}\left(|\mathfrak{z}|^{2} \circ \operatorname{tr}\langle W\rangle\right)$ is continuous. Then $\mathfrak{z} \cdot W \in \mathcal{K}$.

Proof. Lemma 3.8 allows us to confine ourselves to the case $d=1$.

The assumptions of the lemma imply by Theorem I.4.40 [6] existence of the process $M \equiv \mathfrak{z} \cdot W$. The same theorem asserts that $M \in \ell \mathcal{M}_{2}$ and $\langle M\rangle=\mathfrak{z}^{2} \circ\langle W\rangle$, From the last equality we also have by Corollary 3.11 $\mathrm{E}^{0} M^{2}=\mathrm{E}^{0}\left(\mathfrak{z}^{2} \circ\langle W\rangle\right)$, which together with (25) proves property $\mathrm{M1}$ of $M$ and continuity of $\mathrm{E}^{0} M^{2}$.

The relation $M \in \ell \mathcal{M}_{2}$ implies existence of an increasing to infinity sequence $\left(\sigma_{n}\right)$ of stopping times such that for all $n \in \mathbb{N}, t>s \geq 0$

$$
\mathrm{E}\left(M\left(t \wedge \sigma_{n}\right) \mid \mathcal{F}(s)\right)=M\left(s \wedge \sigma_{n}\right) .
$$

Setting in Lemma 3.10 at first $\tau=t \wedge \sigma_{n}$ and then $\tau=t$ and taking to account that $\langle M\rangle$ is an increasing process, we get with account of Lemma 2.3

$\mathrm{E}^{0} M\left(t \wedge \sigma_{n}\right)^{2} \leq \mathrm{E}^{0} M(t)^{2}$, which together with M1 entails stochastic boundedness of the sequences

$\left(\mathrm{E}^{0}\left(M\left(t \wedge \sigma_{n}\right)^{2}\right), n \in \mathbb{N}\right)$ and (in view of Lemma 2.4) $\left(\mathrm{E}\left(M\left(t \wedge \sigma_{n}\right)^{2} \mid \mathcal{F}(s)\right), n \in \mathbb{N}\right)$. So Lemma 2.16 asserts that $\mathrm{E}\left(M\left(t \wedge \sigma_{n}\right) \mid \mathcal{F}(s)\right) \stackrel{\mathrm{P}}{\longrightarrow} \mathrm{E}(M(t) \mid \mathcal{F}(s))$. Thus, letting $n \rightarrow \infty$ in (26), we obtain M2.

\section{The Main Result}

Lemma 4.1. Let $\Lambda$ be a continuous increasing function, $b$ and $H$ be bounded in each interval Borel functions and $U$ be a function satisfying, for all $t \in \mathbb{R}_{+}$, the equality

$$
U(t)=\int_{0}^{t} q(s) \mathrm{d} \Lambda(s)+H(t),
$$

where $q$ is a Borel function with values in $\mathbb{R} \cup\{-\infty\}$ such that $q \leq-b U$. Suppose also that

$$
\int_{0}^{t}|b(s)| \mathrm{d} \Lambda(s)<\infty
$$

for all $t$. Then $U \leq T$, where $T$ is the solution of the equation

$$
T=-(b T) \circ \Lambda+H .
$$

Proof. By condition (28) and the assumptions about $\Lambda$ the integral $b \circ \Lambda$ exists on $\mathbb{R}_{+}$and is a function of locally bounded variation. Equality (27) and the assumptions about $\Lambda$ and $H$ show that $U$ is a Borel function. So $(b U) \circ \Lambda=U \circ(b \circ \Lambda)$. The assumptions of the lemma imply existence of the integral

$q \circ \Lambda(=U-H$ because of (27)), as well (so that $q>-\infty$ almost everywhere w.r.t. the measure with distribution function $\Lambda)$. This entitles us to define the function $h$ by $h=(q+b U) \circ \Lambda$. It decreases, since, by assumption, $q+b U \leq 0$ and $\Lambda$ increases. Also, it is continuous, since so is $\Lambda$.

Denoting $y=U-T$ and subtracting (27) from (29), we get the equation $y=-y \circ(b \circ \Lambda)+h$. Hence, taking to account that $h$ is continuous and starts from zero, we find

$$
y(t)=\mathrm{e}^{-b \circ \Lambda(t)} \int_{0}^{t} \mathrm{e}^{b \circ \Lambda(s)} \mathrm{d} h(s) .
$$

The function $h$ being decreasing, the r.h.s. is nonpositive. $\square$

Corollary 4.2. Let $\Lambda$ be a continuous increasing $\mathbb{F}$-adapted random process, $b$ be an $\mathbb{F}$-progressive random process with values in $\mathbb{R} \cup\{-\infty\}$ satisfying, for all $t$, condition (28), $H$ be an $\mathbb{F}$-semimartingale and $U$ be a random process satisfying, for all $t$, equality (27), where $q$ is a measurable random process such that $q \leq-b U$. Then for all $t$

$$
U(t) \leq \mathrm{e}^{-R(t)} H(0)+\mathrm{e}^{-R(t)} \int_{0}^{t} \mathrm{e}^{R(s)} \mathrm{d} H(s),
$$

where $R=b \circ \Lambda$.

Proof. Denote $V=\mathrm{e}^{-R}$. Noting that $(b T) \circ \Lambda=T \circ R$ and taking to account continuity of $\Lambda$, we write down the solution of (29):

$$
T(t)=H(t)+\mathrm{e}^{R(t)} \int_{0}^{t} \mathrm{e}^{-R(s)} H(s) \mathrm{d} R(s) .
$$

By construction $R$ is a continuous process of locally bounded variation, so $\mathrm{e}^{-R} \mathrm{~d} R=-\mathrm{d} V$. By Proposition I.4.49d [6] the covariation of any such process and a semimartingale equals zero, so the integration-by-parts formula yields $H \circ V=H V-H(0) V(0)-V \cdot H$. Thus $\left(\mathrm{e}^{-R} H\right) \circ R=H(0)-H V+V \cdot H$, which turns (31) into

$$
T(t)=\mathrm{e}^{-R(t)} H(0)+\mathrm{e}^{-R(t)} \int_{0}^{t} \mathrm{e}^{R(s)} \mathrm{d} H(s) .
$$

Now, (30) follows from Lemma 4.1. $\square$

The main result of this article concerns equations of the kind

$$
X(t)=\int_{0}^{t} Q(s, X(s)) \mathrm{d} t(s)+Y(t),
$$


and relies on the assumption

S. For every $\mathbb{R}^{d}$-valued random process $Y \in \mathcal{K}$ equation (32) has a unique strong solution.

As usually, $S^{c}$ signifies the continuous martingale constituent (see $[1,5,6]$ ) of a semimartingale $S$.

Theorem 4.3. Let $l \in \mathcal{V}_{0}^{+, c}, M$ be an $\mathbb{R}^{d}$-valued process of class $\mathcal{K}$ and $Q$ be an $\mathbb{R}^{d}$-valued random function on $\mathbb{R}_{+} \times \mathbb{R}^{d}$, continuous in $x \in \mathbb{R}^{d}$ and $\mathbb{F}$ progressive in $(\omega, t) \in \Omega \times \mathbb{R}_{+}$. Suppose also that condition $\mathbf{S}$ is fulfilled and there exists an $\mathcal{F}(0) \otimes \mathcal{B}_{+}$measurable in $(\omega, t)$ nonnegative random process $\psi$ such that $x^{\mathrm{T}} Q(t, x) \leq-\psi(t)|x|^{2}$ and

$$
\int_{0}^{t} \psi(s) \mathrm{d} t(s)<\infty, \quad t \in \mathbb{R}_{+} .
$$

Then the strong solution of the equation

$$
X(t)=\int_{0}^{t} Q(s, X(s)) \mathrm{d} \imath(s)+M(t)
$$

satisfies, for all $t$, the inequality

$$
\mathrm{E}^{0}|X(t)|^{2} \leq \mathrm{e}^{-\Psi(t)}|M(0)|^{2}+\mathrm{e}^{-\Psi(t)} \int_{0}^{t} \mathrm{e}^{\Psi(s)} \mathrm{dE}^{0} \operatorname{tr}\langle M\rangle(s),
$$

where $\Psi=2 \psi \circ \imath$.

Proof. Denote

$\tau_{n}=\inf \{s:|X(s)| \geq n\}, M_{n}(t)=M\left(t \wedge \tau_{n}\right)$, so that $\tau_{n}$ is a stopping time, $M_{n} \in \ell \mathcal{M}_{2}$ and

$$
|X(s-)| \leq k \quad \text { as } \quad s \leq \tau_{n} .
$$

Let further $X_{n}$ denote the solution of the equation

$$
X_{n}(t)=\int_{0}^{t} Q\left(s, X_{n}(s)\right) \mathrm{d} \imath(s)+M_{n}(t)
$$

(this definition of $X_{n}$ is correct due to condition $\mathbf{S}$ ). Then $X_{n}(s-)=X(s-)$ as $s \leq \tau_{n}$ (because $M_{n}=M$ for these $s$ ). Consequently,

$$
\left(X_{n}^{\mathrm{T}}\right)^{-} \cdot M_{n}=\left(\left(X^{\mathrm{T}}\right)^{-} I_{\left[0, \tau_{n}\right]}\right) \cdot M .
$$

By the choice of $M$ and by Corollary 3.11 and Theorem $3.9 \mathrm{E}^{0} \operatorname{tr}\langle M\rangle(t)<\infty$ for all $t$ and the process $\mathrm{E}^{0} \operatorname{tr}\langle M\rangle$ is continuous. Then because of (35)

$$
\mathrm{E}^{0}\left(\left|X^{-}\right|^{2} I_{\left[0, \tau_{n}\right]}\right) \circ \operatorname{tr}\langle M\rangle(t)<\infty \text { for all } t \text {. Obviously, }
$$

the process $\mathrm{E}^{0}\left(\left|X^{-}\right|^{2} I_{\left[0, \tau_{n}\right]}\right) \circ \operatorname{tr}\langle M\rangle$ is continuous, too.

Thus Lemma 3.12 asserts that $\left(\left(X^{\mathrm{T}}\right)^{-} I_{\left[0, \tau_{n}\right]}\right) \cdot M \in \mathcal{K}$, whence in view of (37)

$$
\mathrm{E}^{0}\left(X_{n}^{\mathrm{T}}\right)^{-} \cdot M_{n}=0 .
$$

Denote

$$
\begin{aligned}
& \varphi_{n}(\tau)=X_{n}(s)^{\mathrm{T}} Q\left(s, X_{n}(s)\right), \\
& D_{n}(t)=2 \int_{0}^{t} \varphi_{n}(s) \mathrm{d} \imath(s),
\end{aligned}
$$

$H_{n}=\left|M_{n}(0)\right|^{2}+\mathrm{E}^{0} \operatorname{tr}\left[M_{n}\right]$. From (36) we have by the assumptions about $\imath$ and $M$

$$
X_{n}^{c}=M_{n}^{c}, \quad \Delta X_{n}=\Delta M_{n}, \quad X_{n}(0)=0 .
$$

By Theorem 2.4.6 in [1] (or, the same, Theorem I.4.47 in [6])

$$
\left[M_{n}\right](t)=\left\langle M_{n}^{c}\right\rangle(t)+\sum_{0<s \leq t} \Delta M_{n}(s) \Delta M_{n}(s)^{\mathrm{T}} .
$$

Writing Itô's formula for $f\left(X_{n}(t)\right)$ and putting $f(x)=|x|^{2}$, so that $f^{\prime}(x)=2 x^{\mathrm{T}},(1 / 2) f^{\prime \prime}=\mathbf{1}$ (a twice covariant tensor), $f(x+y)-f(x)-f^{\prime}(x) y=|y|^{2}$, we get with account of (36), (40) and (41), continuity of $l$ and the identity $|x|^{2}=\operatorname{tr} x x^{\mathrm{T}}$

$$
\left[M_{n}\right](t)=\left\langle M_{n}^{c}\right\rangle(t)+\sum_{0<s \leq t} \Delta M_{n}(s) \Delta M_{n}(s)^{\mathrm{T}} .
$$

By Theorem 3.9 and Corollary $3.11 \mathrm{E}^{0} \operatorname{tr}[M](t)<\infty$ for all $t$ since $M \in \mathcal{K}$. Hence and from the evident inequality $\operatorname{tr}\left[M_{n}\right] \leq \operatorname{tr}[M]$ we have $H_{n}(t)<\infty$, which together with (38) yields, by Lemma 2.11,

$$
\mathrm{E}^{0}\left(2\left(X_{n}^{\mathrm{T}}\right)^{-} \cdot M_{n}+\operatorname{tr}\left[M_{n}\right]\right)=H_{n} .
$$

By construction and the assumptions about $Q$ and $\imath D_{n} \leq 0$, whence by Formula (2) for nonnegative random variables $\mathrm{E}^{0}\left(\left|X_{n}\right|^{2}-D_{n}\right)=\mathrm{E}^{0}\left|X_{n}\right|^{2}-\mathrm{E}^{0} D_{n}$. The last three equalities together with Lemmas 2.11 and 2.13 imply that

$$
\mathrm{E}^{0}\left|X_{n}\right|^{2}=\left|M_{n}(0)\right|^{2}+\mathrm{E}^{0} D_{n}+H_{n} .
$$

By construction and the assumption on $Q$ the process $\varphi_{n}$ is càdlàg and non-positive. Then from (39) we have by the choice of $l$ and by Theorem 2.19

$$
\mathrm{E}^{0} D_{n}=q_{n} \circ \Lambda,
$$

where $q_{n}(t)=\mathrm{E}^{0}\left(X_{n}(t)^{\mathrm{T}} Q\left(t, X_{n}(t)\right)\right), \Lambda=2 \imath$. Then equality (42), whose 1.h.s. is, evidently, an $\overline{\mathbb{R}}_{+}$-valued process, together with established above finiteness of $H_{n}$ shows that $q_{n} \circ \Lambda(t)>-\infty$ for all $t$ (though $q_{n}$ may take the value $-\infty$ with positive probability).

By the construction of $q_{n}$, the assumption on $Q$ and by Lemma $2.3 q_{n} \leq-\mathrm{E}^{0}\left(\psi\left|X_{n}\right|^{2}\right)$. The process $\imath$ was assumed increasing and therefore $\Lambda$ increases, too; the process $\psi$ was assumed nonnegative, so $q_{n} \leq 0$ by Lemma 2.3. Thus $q_{n} \circ \Lambda \leq 0$, which together with (42), (43) and finiteness of $H_{n}$ yields $\mathrm{E}^{0}\left|X_{n}\right|^{2}<\infty$. Then from $\mathcal{F}(0)$-measurability of $\psi(t), t \in \mathbb{R}_{+}$, we have by Lemma $2.15 \mathrm{E}^{0}\left(\psi\left|X_{n}\right|^{2}\right)=\psi \mathrm{E}^{0}\left|X_{n}\right|^{2}$ and therefore $q_{n} \leq-\psi \mathrm{E}^{0}\left|X_{n}\right|^{2}$. From this inequality and (33), (42), (43) we get by Corollary 4.2

$$
\mathrm{E}^{0}\left|X_{n}(t)\right|^{2} \leq \mathrm{e}^{-\Psi(t)}\left|M_{n}(0)\right|^{2}+\mathrm{e}^{-\Psi(t)} \int_{0}^{t} \mathrm{e}^{\Psi(s)} \mathrm{d} H_{n}(s)
$$


and all the more

$$
\begin{aligned}
& \mathrm{E}^{0}\left|X_{n}(t)\right|^{2} \leq \mathrm{e}^{-\Psi(t)}\left|M_{n}(0)\right|^{2} \\
& +\mathrm{e}^{-\Psi(t)} \int_{0}^{t} \mathrm{e}^{\Psi(s)} \mathrm{dE}^{0} \operatorname{tr}[M](s) .
\end{aligned}
$$

Obviously, $X_{n}(t) \rightarrow X(t), M_{n}(0) \rightarrow M(0)$ as $n \rightarrow \infty$. Then Corollary 2.8 asserts that

$\mathrm{E}^{0}|X(t)|^{2} \leq \underline{\lim }^{0}\left|X_{n}(t)\right|^{2}$. It remains to note that

$\mathrm{E}^{0} \operatorname{tr}[M]=\mathrm{E}^{0} \operatorname{tr}\langle M\rangle$ by Corollary 3.11 .

\section{REFERENCES}

[1] I. I. Gikhman and A. V. Skorokhod, "Stochastic Differential Equations and Their Applications," Naukova Dumka,
Kiev, 1982.

[2] A. N. Shiryaev, "Probability," Springer, Berlin, 1996.

[3] R. Z. Khasminsky, "Stochastic Stability of Differential Equations," 2nd Edition, Springer, Berlin, 2012. doi:10.1007/978-3-642-23280-0

[4] L. J. Shen and J. T. Sun, " $p$-th Moment Exponential Stability of Stochastic Differential Equations with Impulse Effect," Science China Information Sciences, Vol. 54, No. 8, 2011, pp. 1702-1711. doi:10.1007/s11432-011-4250-7

[5] R. Sh. Liptser and A. N. Shiryaev, "Theory of Martingales," Kluwer, Dordrecht, 1989. doi:10.1007/978-94-009-2438-3

[6] J. Jacod and A. N. Shiryayev, "Limit Theorems for Stochastic Processes," Springer, Berlin, 1987. 